associated with multiple foodborne outbreaks throughout the world, mostly attributed to pig and pig products. Recently, we have demonstrated that two distinct groups of Salmonella 4,[5],12:i:circulate in the USA and Europe, with the majority of isolates recovered during recent years belonging to an emerging multidrug-resistant clade (Elnekave et al. 2018). We applied Bayesian phylodynamic reconstruction to uncover the evolutionary history of this clade. We used a dataset of wholegenome sequences of 1446 4,[5],12:i:- isolates from different sources (livestock, human, food products, and others) from the USA $(n=752)$ and Europe $(n=694)$, collected between 2008 and 2017 and belonging to the Multilocus Subtype 34, which was predominant in the emerging clade (Elnekave et al. 2018). A subset $(n=110)$ of Salmonella 4,[5],12:i: isolates was then randomly selected after stratifying by location and year of isolation in order to achieve balanced sampling. Evidence of temporal signal was confirmed by looking at root-to-tip divergences using TempEst. Evolutionary hypotheses using strict and relaxed-clock models were tested using BEAST for a variety of demographic models and assuming a general time reversible substitution model. Model selection was performed by estimating Bayes Factors using path sampling and stepping-stone sampling. The selected model was then used for applying discrete trait models comparing different scenarios of transmission between locations (i.e. bidirectional symmetric/asymmetric or unidirectional). Our preliminary phylodynamic inference results indicate that the origin of this subtype was in Europe and dates back to 1990 (HPD 95\%: 1984 2001). We report an exponential growth rate of 0.362 per year, which corresponds to a doubling time of 1.43 years. Our results suggest that this subtype was introduced to the US in the year 2000 (HPD 95\%: 1994-2006). Phylodynamic analysis suggests that the recent increase in isolation of Salmonella 4,[5],12:i:- from different sources in the USA may be due to the exponential expansion of an emerging clone which originated in Europe and then expanded to the USA. The emergence and expansion of this serovar is of great public health importance due to the high prevalence of multidrug resistance traits found in USA isolates from this group and especially due to the presence of plasmidmediated resistance genes for quinolones and extended spectrum cephalosporins, key antimicrobials used for the treatment of invasive Salmonella infections.

A67 Bloodstream infections by carbapenem-resistant Klebsiella pneumoniae subsp. pneumoniae: Bayesian phylogenetic analysis of whole genomes

Eleonora Cella, ${ }^{1}$ Davide Leoni, ${ }^{2}$ Walter Mirandola,${ }^{3}$ Carla Fontana,${ }^{3}$ Loredana Sarmati, ${ }^{2}$ Massimo Andreoni, ${ }^{2}$ Silvia Angeletti, ${ }^{4}$ and Massimo Ciccozzi ${ }^{1}$

${ }^{1}$ Unit of Medical Statistic and Molecular Epidemiology, University Campus Bio-medico of Rome, Rome, Italy, ${ }^{2}$ Clinical Microbiology Laboratories, Polyclinic of Tor Vergata, Viale Oxford 81, Rome 00133, Italy, ${ }^{3}$ Clinical of Infectious Diseases, Department of System Medicine, Tor Vergata University, Rome, Italy and ${ }^{4}$ Unit of Clinical Laboratory Science, University Campus Bio-medico of Rome, Rome, Italy

Bloodstream infection (BSI) caused by carbapenemase-producing Enterobacteriaceae (CPE) is a major public health concern, particularly in the hospital setting. The rapid detection of resistance patterns is of paramount importance for establishing the proper antibiotic regime. In addition, in countries where CPE are endemic, it is also important to evaluate genetic relationship among the isolates in order to trace pathogen circulation and to improve the infection control programs. This study is an application of a rapid blood culture (BC) workflow consisting of fast reporting of Gram stain results, rapid pathogen identification (using MALDI TOF technology), and a molecular assay for the detection of the major genes conferring resistance, all of them performed directly from positive BCs. The application of phylogenetic and phylodynamic analyses to bacterial wholegenome sequencing (WGS) data have become essential in the epidemiological surveillance of multidrug-resistant nosocomial pathogens. We analyzed 40 strains of Klebsiella pneumoniae subsp. pneumoniae (KP) carrying blaKPC (KP-KPC), randomly selected among 147 CPE identified from BCs collected from consecutive patients from 2013 to 2016 . The number of BSIs-related CPE were $23,31,43$, and 50 in 2013, 2014, 2015, and 2016, respectively Among 147 CPE isolates, 143 were KP and four were Escherichia coli (EC). The gene blaKPC was detected in 117 strains of KP and in four strains of EC. Other carbapenemase genes, such as blaVIM and blaOXA-48, were detected in four and nine different isolates of KP, respectively. Moreover, $13 \mathrm{KP}$ strains carried two resistance genes: twelve vehicled blaKPC plus blaVIM and one blaKPC plus blaOXA48. Phylogenetic analysis of bacterial WGS data was used to investigate the evolution and spatial dispersion of KP in support of hospital infection control. The maximum likelihood tree showed two main clades statistically supported, with statistical support for several subclusters within as well. The minimum spanning tree showed mixing between sequences from different years and wards with only few specific groups. Bayesian analyses are ongoing, as the aid of Bayesian genomic epidemiology in combination with active microbial surveillance is highly informative regarding the development of effective infection prevention in healthcare settings or constant strain reintroduction.

A68 Characterization and comparative genomic analysis of two Bacillus megaterium lytic bacteriophages

A. Sharaf, ${ }^{1}$ M. Oborník, ${ }^{2,3}$ A. Hammad, ${ }^{4}$ S. El-Afifi, ${ }^{5}$ and E. Marei ${ }^{5}$

${ }^{1}$ Genetic Department, Faculty of Agriculture, Ain Shams University, Cairo, Egypt, ${ }^{2}$ Biology Centre CAS, Institute of Parasitology, České Budějovice, Czech Republic, ${ }^{3}$ Faculty of Science, University of South Bohemia, České Budějovice, Czech Republic, ${ }^{4}$ Department of Microbiology, Faculty of Agriculture, Minia University, Minia, Egypt and ${ }^{5}$ Department of Agricultural Microbiology, Virology Laboratory, Ain Shams University, Cairo, Egypt

Next-generation sequencing technologies provide unique possibilities for the comprehensive assessment of the environmental diversity of bacteriophages. Many Bacillus bacteriophages have been isolated, but very few Bacillus megaterium bacteriophages have been characterized. Here, we describe biological characteristics and whole-genome sequences and their annotations for two new isolates of the B. megaterium bacteriophages (BM5 and BM10), isolated from Egyptian soil samples and representing two different groups according to their host range and amplified fragment length polymorphism profiles Both phages have been displaying different thermal inactivation points $\left(82\right.$ and $\left.78^{\circ} \mathrm{C}\right)$ and $\mathrm{pH}$ tolerance range $(5-9.2$ and $5-8.4 \mathrm{pH})$ while having the same longevity in vitro (192 h). Electron microscopy observation has proved that both phages belonged to the Myoviridiae family. Furthermore, growth analyses indicated that phages BM5 and BM10 have a shorter latent period (20 and $25 \mathrm{~min}$ ) and smaller burst size (103 and $117 \mathrm{PFU}$ ) than is typical for Bacillus phages. The genome sizes of phages BM5 and BM10 were $165,031 \mathrm{bp}$ and $165,213 \mathrm{bp}$, respectively, with a modular organization. Bioinformatic analyses of BM5 and BM10 genomes enabled assignments of putative functions to 97 and 65 putative ORFs, respectively. Comparative analysis of BM5 and BM10 genome structures with other $B$. megaterium bacteriophages revealed relatively high levels of sequence and organizational identity. Both genomic comparisons and phylogenetic analyses support the conclusion that the sequenced phages (BM5 and BM10) belong to different sub-clusters (L5 and L7) within L cluster and display different lifestyles (lysogenic and lytic). Sequenced phages encode proteins associated with Bacillus pathogenesis. BM5 does not contain any tRNA sequences, while BM10 genome codes for 17 tRNAs.

\section{A69 Phylodynamics of language evolution}

\section{K. Hoffmann and D. Kühnert}

Max Planck Institute for the Science of Human History, Jena, Germany

We used Bayesian evolutionary analysis to study linguistic data and infer phylogenetic trees of language evolution. Languages were encoded as binary strings indicating the presence or absence of members of cognate classes, the equivalence of classes of words with similar meaning, and shared ancestry. These strings formed the alignment data used to compute the posterior likelihood of a tree with respect to Bayes' formula. Informative priors are crucial for testing hypotheses regarding the age of common ancestry and divergence times and should include as much available information as possible. Here, we investigated the birth-death process as a method to construct tree priors specifically suitable for modeling the evolution of cognate data. To test these models, we will use a dataset of the languages from Vanuatu, an island nation featuring world's highest language density. 